\title{
MORBILIDAD Y MORTALIDAD DE LOS SOLDADOS DEL EJÉRCITO ESPAÑOL, 1886-1933 ${ }^{1}$
}

\author{
Roser Nicolau \\ Dpto. de Economía e Ha Económica-Universidad Autónoma de Barcelona \\ roser.nicolau@uab.cat \\ Pedro Fatjó \\ Dpto. de Economía e Ha Económica-Universidad Autónoma de Barcelona \\ pedro.fatjo@uab.cat
}

Recibido: 20 enero 2015; Aceptado: 20 julio 2015.

Cómo citar este artículo/Citation: Nicolau, Roser y Pedro Fatjó (2016), “Morbilidad y mortalidad de los soldados del Ejército español, 1886-1933", Asclepio 68 (1): p129 doi: http://dx.doi.org/10.3989/asclepio.2016.08

RESUMEN: Este artículo presenta los primeros resultados de un proyecto de investigación sobre la salud de los soldados del Ejército español, en época de paz, entre 1860 y 1936, con especial atención a la mortalidad y la morbilidad y a la tipología de las enfermedades con mayor incidencia en este colectivo. Aquí se abordan dos objetivos de naturaleza metodológica: en primer lugar, una introducción a las posibilidades y limitaciones de las fuentes históricas sanitarias militares para el estudio de la salud del colectivo militar; y, en segundo lugar, un análisis de la evolución de los efectivos y las características del colectivo masculino en edad de recluta y su grado de representatividad respecto a toda la población masculina del mismo grupo de edad. Así mismo, se avanzan unos primeros resultados provisionales sobre algunos indicadores de mortalidad y morbilidad de los soldados y de la población civil que pueden estimarse a partir de las fuentes sanitarias militares.

PALABRAS CLAVE: Morbilidad; Mortalidad; Ejército; Población; España.

MORBIDITY AND MORTALITY OF THE SOLDIERS OF THE SPANISH ARMY, 1886-1933

ABSTRACT: This article presents the first results of a research project aimed at studying the state of health of Spanish army troops in peacetime between 1860 and 1936 . Special attention is paid to mortality, morbidity, and the typology of the diseases which had the greatest impact on this group. Two methodological objectives are pursued: first, an introduction to the possibilities and limitations of historical sanitary records for the study of the state of health of the military class is carried out; second, the number of military recruits and their collective features as a group, and to what degree this group is representative of their age and gender group as a whole, are examined. Similarly, the initial results on some indicators of mortality and morbidity among both the drafted troops and the civilian population, as reflected in sanitary records, are also set forth.

KEY WORDS: Morbidity; Mortality; Army; Population; Spain.

Copyright: (C) 2016 CSIC. Este es un artículo de acceso abierto distribuido bajo los términos de la licencia Creative Commons Attribution (CC BY) España 3.0. 
El estudio de la salud de los soldados en los ejércitos en época de paz ofrece particular interés, puesto que se centra en un colectivo humano bien delimitado en cuanto a sexo y edad, y que requiere la consideración de múltiples variables: el sistema de selección y reclutamiento y su relación con la salud de los reclutas antes de entrar en filas; las condiciones de alojamiento e higiene colectiva en que vivirán durante el servicio activo y la incidencia de enfermedades infecciosas; la alimentación y su relación con la salud; el papel de los servicios sanitarios militares; la entidad de los recursos presupuestarios que determinaban las condiciones de vida durante la duración del servicio, etc. En el presente trabajo abordaremos algunos aspectos específicos que forman parte de una investigación más amplia sobre la salud de los soldados del ejército español a lo largo del periodo 1860-1936.

\section{INTRODUCCIÓN A LAS FUENTES SANITARIAS MILITARES}

Las estadísticas militares de naturaleza sanitaria, pueden proporcionar información sobre la evolución de la salud de la población; una información distinta y, en ocasiones, más completa que la que se encuentra en las estadísticas de mortalidad y morbilidad del conjunto de la población. Su principal limitación es que la información afecta sólo a un grupo en particular de este colectivo masculino, el de edad de reclutamiento (alrededor de los 21 años para el período estudiado), si bien permite conocer con notable precisión las cifras de mortalidad y de morbilidad del grupo, así como el papel de determinadas enfermedades y su evolución a lo largo del tiempo.

La estadística militar fue pionera en España en la elaboración de series estadísticas de naturaleza sanitaria, yendo por delante de la estadística civil durante buena parte del siglo XIX. Aunque no hemos podido establecer con total precisión el inicio de la publicación de series regulares de estadísticas sanitarias del Ejército o de la Marina, las primeras que hemos podido consultar datan de la segunda mitad de la década de 1860 y estaban incluidas en los dos Anuarios Estadísticos de España publicados en esos años; ${ }^{2}$ entonces se centraban en cuantificar el movimiento de enfermos en los hospitales militares y cívico-militares, si bien la clasificación por enfermedades era muy genérica y poco depurada. Estos primeros datos estadísticos abarcaban a todo el personal atendido en hospitales militares o dependientes del Ministerio de la Guerra, lo que incluía no sólo al Ejército sino a otros cuerpos armados como la Guardia Civil y los Carabineros, además de agregar en los cómputos a civiles, sin que la presentación de los datos permita diferenciar entre unas $u$ otras procedencias. ${ }^{3}$

Es a partir de 1886 cuando se inicia la publicación regular de series sanitarias anuales dotadas de un contenido más homogéneo: son las denominadas Memorias-Resúmenes o Resúmenes de la Estadística Sanitaria del Ejército. Hemos podido localizar análisis comentados de las mismas en la Revista de Sanidad Militar desde dicho año de 1886 y desde 1896 hemos podido utilizar las publicaciones originales. También se encuentran estadísticas sanitarias militares en los Anuarios Estadísticos de España publicados en el primer tercio del siglo XX, entre 1914 y 1935, cuyo contenido procedía de las series militares, pero en ocasiones es incompleto y no siempre homogéneo, de forma que lo mejor es consultar y contrastar ambas fuentes a fin de cubrir las lagunas temporales o de complementar los datos de una o de otra. ${ }^{4}$

Aunque los esfuerzos por elaborar una estadística sanitaria en el Ejército español obedecieron a necesidades propias, no cabe duda que el impulso proporcionado por la Comisión Internacional para la Unificación de la Estadística Sanitaria de los Ejércitos a través de congresos periódicos, fue decisivo. En la conferencia de Budapest de 1894 se acordó por primera vez la adopción de un formulario común a todos los ejércitos de los estados participantes. Sucesivas conferencias celebradas en Moscú (1897), París (1900), Madrid (1903) y Berlín (1907) introdujeron diversas modificaciones en el formulario. En el caso español, un primer modelo se puso en marcha en 1903, seguido por el de 1907, que se mantuvo vigente hasta 1936 (Rodríguez-Ocaña, 1992, p. 48).

Las variables cuantificadas en estas publicaciones se expresaban tanto en valores absolutos como proporcionales (\%) y hacían referencia a la mortalidad y a la morbilidad: total de ingresados y muertos por las calificadas como "enfermedades principales", los días totales de estancia de todos los ingresados de cada enfermedad y la duración media de los días de hospitalización por enfermo ingresado. La mortalidad hacía referencia a los fallecimientos que habían tenido lugar en hospitales y enfermerías militares, excluyéndose del cómputo los suicidios, los accidentes y los muertos o heridos en combate. Por morbilidad (morbosidad en las fuentes del siglo XIX), se contabilizaban los enfermos ingresados en hospitales militares, excluyendo a los ingresados en las enfermerías de los acuartelamientos; tampoco se incluían a los accidentados o heridos en combate.

En cuanto al área geográfica que cubrían las estadísticas sanitarias militares, hasta finales del siglo XIX 
diferenciaban entre Península e Islas Adyacentes, por un lado y Ultramar (Cuba, Puerto Rico y Filipinas), por otro; con la pérdida de las colonias el marco geográfico será el de la Península e Islas Adyacentes y desde 1909 se individualizarán las guarniciones africanas. En este último periodo, de 1909 a 1936, las memorias-resumen no permiten diferenciar entre las cifras peninsulares y las del Protectorado. Si bien es cierto que la lectura de diversos artículos de la Revista de Sanidad Militar hace posible la identificación de las enfermedades que tenían una mayor incidencia entre las tropas destinadas en África, como sucedía con el paludismo por ejemplo, no podemos saber la incidencia en cada uno de ambos escenarios geográficos.

La relación de las enfermedades registradas en las series estadísticas castrenses experimentó variaciones a lo largo del tiempo, dirigidas hacia una mayor precisión en su identificación. En los años de 1860 la ordenación era muy simple, vertebrada en dos bloques: enfermedades de cirugía y enfermedades de medicina; registrándose en el primer grupo una decena de "afecciones" y una veintena en el segundo. La clasificación de las enfermedades de cirugía era muy genérica: "tumores", "hérnicas" y "úlceras"; las lesiones fruto de accidentes ("heridas", "fracturas" y "dislocaciones") eran registradas como si se tratase de enfermedades. La tipología en el caso de la medicina era más extensa pero poco precisa: sirva de ejemplo el caso de las "calenturas", subdivididas en inflamatorias, gastro-biliosas, exantemáticas, catarrales, tifoideas, intermitentes simples e intermitentes malignas. ${ }^{5}$ En la década de 1880 se altera el catálogo de enfermedades, que se agruparán en once categorías: las de medicina y cirugía, que ya no se detallan, y viruela, sarampión, tuberculosis, oftalmológicas, venéreo, sarna, dermatosis, entre otras (Anónimo, 1888, pp. 372-373). De forma progresiva a lo largo de la segunda mitad de la década de 1890 se adoptará la nomenclatura acordada en los sucesivos congresos internacionales de sanidad militar, culminado con la de 1903 que con puntuales modificaciones se mantendrá en vigencia hasta $1936^{6}$. Se trataba de un listado de 38 enfermedades, desde las que estaban mejor definidas en esa época (tuberculosis pulmonar, sífilis, gonorrea, escarlatina, tifus abdominal, viruela, disentería, meningitis, etc.), hasta dolencias muy variadas y poco precisas (enfermedades mentales, oculares, dermatológicas, urinarias, auditivas, etc.) ${ }^{7}$.

De todas formas, pese a esta clase de limitaciones es posible hacer un seguimiento de la incidencia de algunas enfermedades en términos de mortalidad y de morbili- dad, incluyendo los días totales de hospitalización que provocaron cada una de ellas. Aunque no es el objeto específico de este artículo, hemos recopilado información sobre la incidencia de enfermedades respiratorios especialmente virulentas (tuberculosis pulmonar, bronquitis aguda, gripe, neumonía y pleuritis), y también sobre la fiebre tifoidea (llamado en las fuentes como tifus abdominal) ${ }^{8}$, la viruela, el paludismo y la meningitis.

Pero junto a los datos de mortalidad y de morbilidad que hemos visto, las estadísticas sanitarias militares también proporcionan información sobre los jóvenes declarados inútiles por motivos de enfermedad o por defectos físicos, excluyendo los clasificados como cortos de talla, de los que no nos ocuparemos. El seguimiento de esta información no está exento de complicaciones, puesto que las sucesivas leyes de reclutamiento y reemplazo fueron introduciendo modificaciones en los cuadros de causas de exención por inutilidad física, por lo que es necesario enmarcar siempre las cifras en el contexto de esos cambios. Pero una vez tenida en cuenta dicha prevención son una fuente destacada sobre el estado de salud de la población masculina en edad militar antes de ingresar en el ejército.

Además de las estadísticas sanitarias militares, una fuente imprescindible para el estudio de la morbilidad y la mortalidad en el ejército español es la constituida por las revistas especializadas en sanidad militar. Esta clase de publicaciones proporcionan abundante información, en su mayor parte de naturaleza cualitativa pero también ofrecen datos cuantitativos que pueden complementar los obtenidos en las estadísticas o cubrir lagunas de dichas series. La información que definimos como cualitativa es de alcance desigual, pero con frecuencia muy útil para contextualizar y matizar los datos estadísticos y permitir una interpretación más precisa de la evolución de los indicadores demográficos militares. En la década de 1860 surgieron las primeras revistas, aunque todas fueron de corta vida, y a partir de 1875 hay una casi total continuidad en las sucesivas publicaciones que se prolongará hasta 1936.

La revista más antigua es la Revista de Sanidad Militar Española y Extranjera, que inició su andadura en 1864 y desapareció a finales de 1865. Fue seguida por la Revista de Sanidad Militar y General de Ciencias Médicas, que sólo se publicó a lo largo del año 1866. Al año siguiente y también con una duración limitada a un año, apareció la Revista General de Ciencias Médicas y de Sanidad Militar. No será hasta entrada la década de 1870 cuando se reemprenda la edición de una revista especializada con la Gaceta de Sanidad Militar, que se publicará quincenalmente hasta noviembre de 1885 . 
Después de un año sin ninguna revista, en Enero de 1887 verá la luz la Revista de Sanidad Militar, que se mantuvo hasta 1906 y fue reemplazada por la Revista de Sanidad Militar y la Medicina Militar Española, que permanecerá hasta 1910, siendo a su vez substituida por la segunda época de la Revista de Sanidad Militar, la de más larga vida de toda esta clase de publicaciones, pues se editó sin interrupciones desde Enero de 1911 hasta el estallido de la Guerra Civil en $1936^{9}$.

No hemos entrado en una explotación sistemática de estas fuentes bibliográficas contemporáneas, ya que la gran variedad de temas abordados las hacen de gran interés para muy distintas líneas de investigación, por ejemplo, para la historia de la medicina (tratamientos de heridas de armas de fuego o de fracturas, técnicas quirúrgicas, profilaxis, equipamiento hospitalario, instrumental quirúrgico, etc.), pero sí lo hemos hecho con los artículos más directamente relacionados con la mortalidad y morbilidad generales o con alguna de las enfermedades más extendidas entre la tropa, como la viruela o la tuberculosis. La opinión de los médicos militares es muy relevante para aproximarse a la problemática sanitaria que rodeaba la vida de los soldados durante la prestación del servicio, y con frecuencia, expresa una mirada muy crítica con las condiciones higiénico-sanitarias que envolvían la vida de los soldados españoles: las deficiencias higiénicas de muchos acuartelamientos que estaban en directa relación con la amplia difusión de las fiebres tifoideas entre las tropas (Sánchez Fernández, 1907, pp. 42-50); la baja extracción social de la mayoría de los reclutas como una variable destacada para explicar los estragos ocasionados por muchas enfermedades a finales del siglo XIX (Deleito, 1902, pp. 109-114); la mortalidad provocada por la viruela era escandalosamente alta en comparación con otros ejércitos europeos (Martínez Pacheco, 1890, pp. 3-24), eran temas abordados en estas revistas, con una claridad y contundencia que sorprende en publicaciones militares oficiales y de difusión pública.

\section{CARACTERÍSTICAS Y REPRESENTATIVIDAD DEL COLECTIVO}

El propósito es examinar la representatividad de la población sobre la que se establecieron anualmente las estadísticas sanitarias militares. Para ello vamos a partir de la serie anual de los nacimientos que disponemos en España desde 1858 y que permite conocer los efectivos iniciales de las sucesivas generaciones masculinas. A partir de estos efectivos iniciales vamos a estimar que proporción de los varones alcan- zaba la edad de reclutamiento y que proporción de ellos era finalmente observada y sus datos recogidos por las estadísticas militares.

Como muestra el gráfico 1 , el número de nacimientos masculinos aumentó entre las décadas de 1850 y 1880 y desde entonces osciló alrededor de los 335.000, hasta la década de 1970. A partir de dicho año se inició un fuerte descenso que se prolongó hasta fines de siglo. Antes de este descenso, los nacimientos masculinos estuvieron por debajo de los 315.000 anuales en 1919 , en $1937-39$ y en casi todos los años comprendidos entre 1940 y 1957. Las fluctuaciones de la natalidad dejan una huella más duradera en las series porque repercuten en el número de jóvenes adultos y potenciales padres alrededor de treinta años más tarde. La primera caída de los nacimientos, que se produce a lo largo de la década de 1910 y que se agudizó con la epidemia de gripe de 1918, explica sin embargo solo una parte de la caída de los nacimientos después de la guerra. Las generaciones de la década de 1910, mas vacías, padecieron especialmente la mortalidad durante la Guerra Civil, lo que repercutió sin duda en la caída de los nacimientos después de la guerra, pero a este factor se sumó también otro muy importante que fue el descenso muy significativo de la fecundidad durante los años cuarenta y hasta finales de los años cincuenta, en que se inició un pequeño baby boom.

Los varones no eran objeto de interés militar hasta que cumplían una determinada edad que para la mayor parte de las generaciones del siglo XX fue los 21 años. Evidentemente, la proporción de nacidos que alcanzaron aquella edad varió de forma muy significativa en el transcurso del tiempo debido al descenso y a las fluctuaciones de la mortalidad. Las estadísticas del movimiento natural nos han permitido estimar para todas las generaciones del siglo XX y solo para algunas de la segunda mitad del XIX -las de 1858-70 y 1878-82 y representadas todas en el gráfico 2 - sus respectivos niveles de la mortalidad antes de los 2 años de edad y estimar así la proporción de sobrevivientes sobre 1000 nacidos que alcanzaron aquella edad y que sobrevivieron por tanto al segundo aniversario. La tesis doctoral de A. Blanes (2007), que ha reconstruido las tablas de mortalidad de las generaciones nacidas a partir de 1911, y a quien agradecemos que nos haya permitido hacer uso de sus resultados antes de ser publicados, amplía y enriquece de forma muy importante el conocimiento que teníamos hasta ahora de la evolución de la mortalidad española. Sus tablas de mortalidad nos permiten conocer la proporción de sobrevivientes a los 2 años, pero también a los 21 años de las generacio- 
Gráfico 1. Nacimientos de sexo masculino en España, 1858-2010

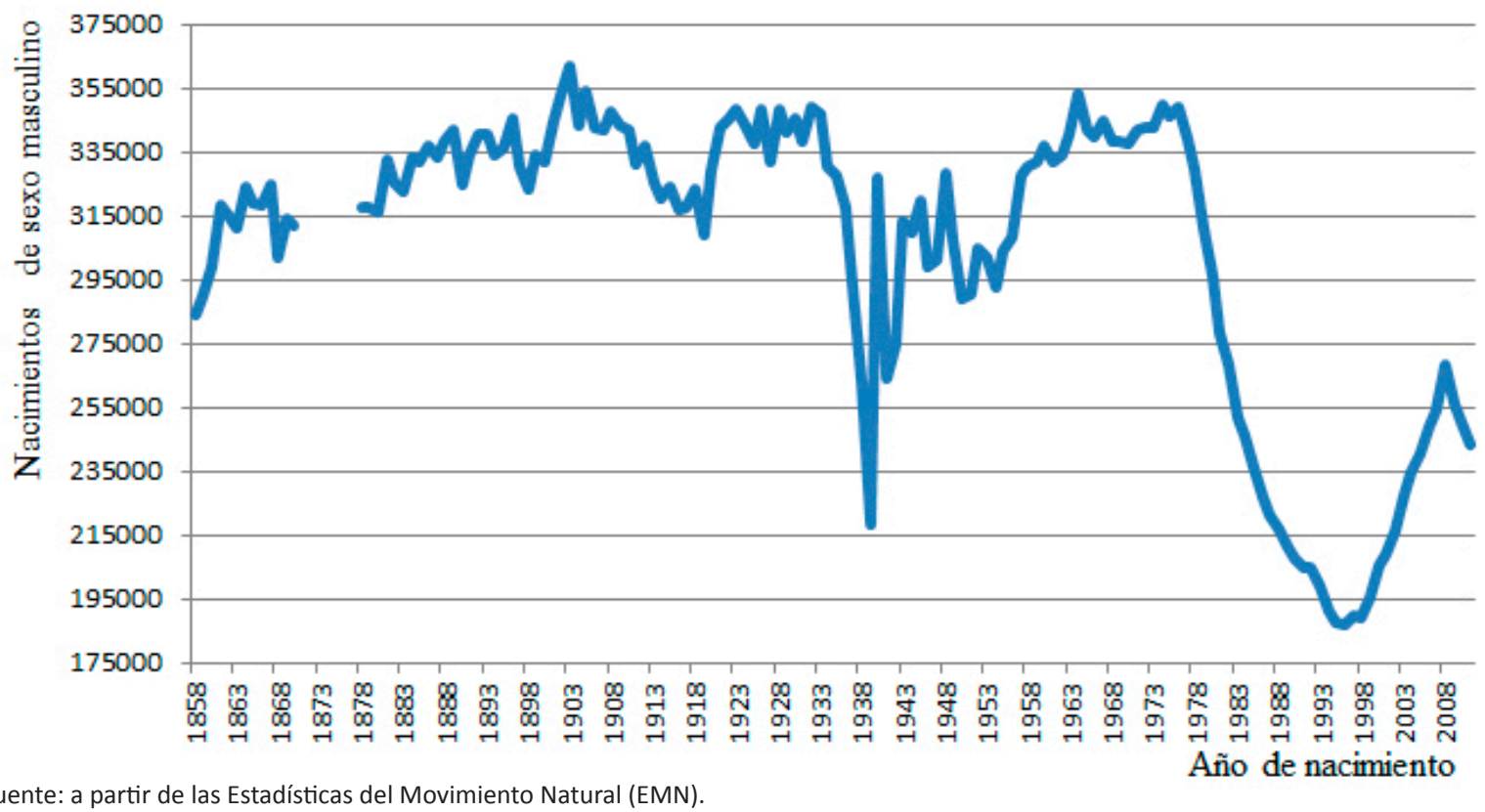

nes masculinas nacidas entre 1911 y 1984 . A partir de la relación observada entre los sobrevivientes a los 2 y los 21 años de las generaciones 1911-1984, hemos ajustado una función con un coeficiente de determinación muy elevado $(R 2=0,995)$ que indica que el ajuste es bueno, lo que no es de extrañar porque la mortalidad antes de los 2 años es un componente importante de la mortalidad antes de los 21 años y porque está muy relacionada también con la mortalidad de 2 a 21 años. Con esta función, representada en el gráfico 2, hemos estimado la proporción de sobrevivientes a los 21 años para las generaciones nacidas antes de 1911, de $1858-70,1878-82$ y $1900-10-$, para las que solo conocíamos las proporciones de sobrevivientes a los 2 años de edad. El gráfico 3 muestra la evolución de estos dos indicadores de la mortalidad de las generaciones.

Los datos disponibles para las generaciones de 185870 y $1878-82$ no muestran un descenso de la mortalidad y, por tanto, un aumento de la proporción de sobrevivientes. La interrupción de los datos de 1883 a 1899 no permite valorar el bajo nivel observado para la generación de 1900, distinguiendo si es un valor representativo también de algunas de las generaciones que la precedieron o si por el contrario fue el resultado de un aumento coyuntural de la mortalidad de la generación de 1900. Dos razones para decantarse por la segunda posibilidad son que los indicadores de la mortalidad por ahora disponibles para aquel periodo y en particular la tasa bruta de mortalidad muestra un descenso a partir de finales de la década de 1880 o principios de la década de 1890 y la segunda razón, es que las series continuas de otros países próximos, como por ejemplo Italia, muestran un aumento coyuntural de la mortalidad de 0 a 2 años para la generación de 1900 (Pérez-Moreda, 2015) ${ }^{10}$. En cualquier caso, para bastantes generaciones de la segunda mitad del XIX, la mortalidad antes de los 2 años alcanzaba a $300 \%$ nacidos y antes de los 21 años había eliminado a la mitad de sus efectivos. Las series continúas para las generaciones del siglo XX revelan en primer lugar el progresivo aumento de la proporción de sobrevivientes a los dos años de edad, que de $700 \%$ en muchas generaciones del siglo XIX y en la de 1900, alcanza la proporción de $800 \%$ en la generación de 1912, 900\%。 en la de 1947 y $995 \%$ on la de 1997.

Hay dos grupos de generaciones en el siglo XX que no registran una mejora en sus proporciones de sobrevivientes a los 2 años de edad y algunas de ellas un significativo descenso: el primer grupo es el de las generaciones de 1913 a 1921 y el segundo, las generaciones de 1936 a 1941 . El aumento de la mortalidad infecciosa en la segunda década del siglo XX y durante la Guerra Civil e inmediata posguerra explica en gran medida aquellos retrocesos. La sobremortalidad de aquellos dos periodos, y en el segundo no solo por enfermedades infecciosas, afectaron también a los 
Gráfico 2. Relación entre las proporciones de sobrevivientes a los 2 y a los 21 años de las generaciones masculinas españolas, 1911-1984

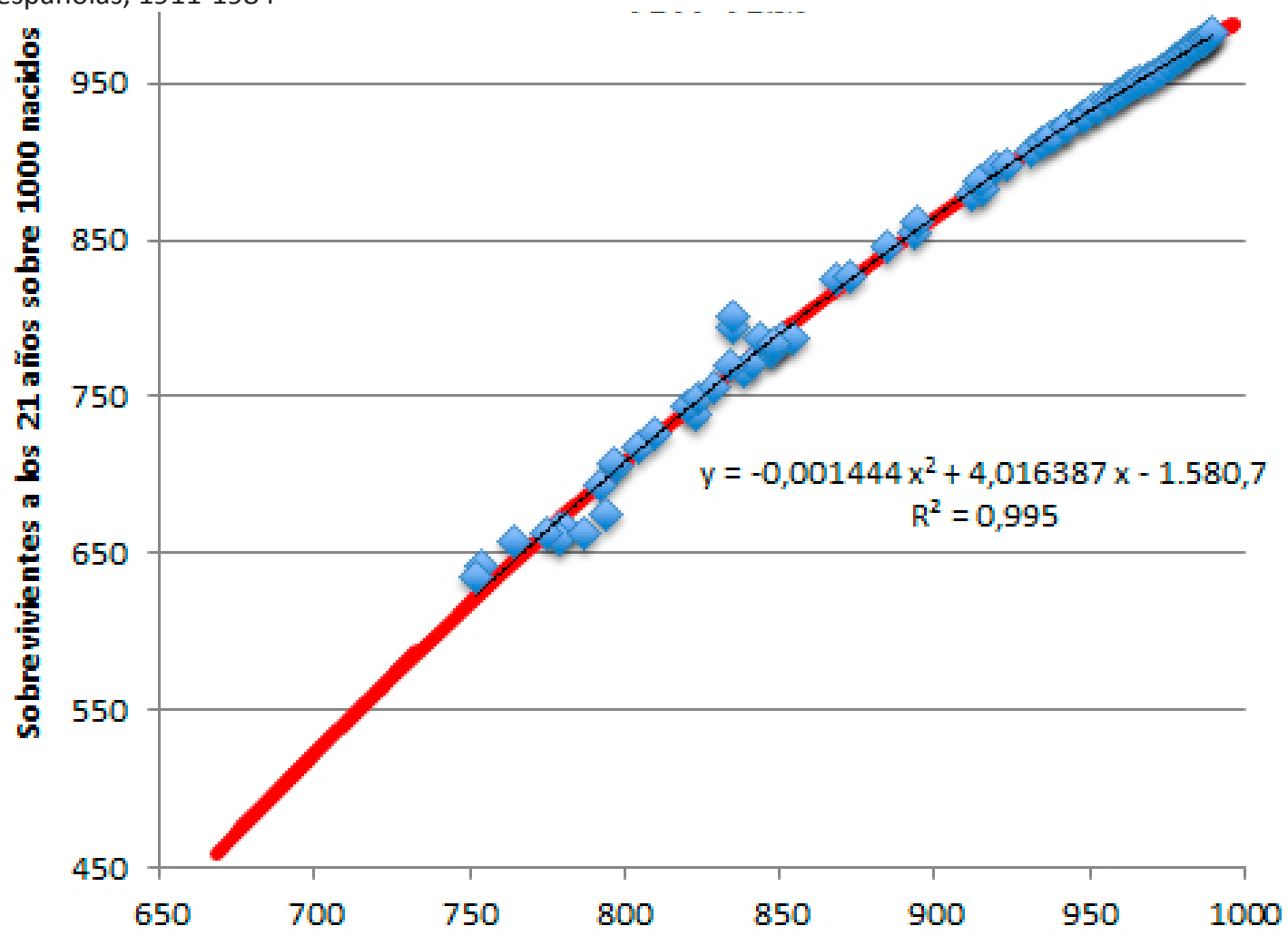

Sobrevivientes a los 2 años sobre 1000 nacidos

Fuente: elaboración propia a partir de las tablas de mortalidad de las generaciones.

Gráfico 3. Proporción de sobrevivientes a los 2 y a los 21 años de edad (sobre 1000 nacidos) de las generaciones masculinas españolas, 1858-2009

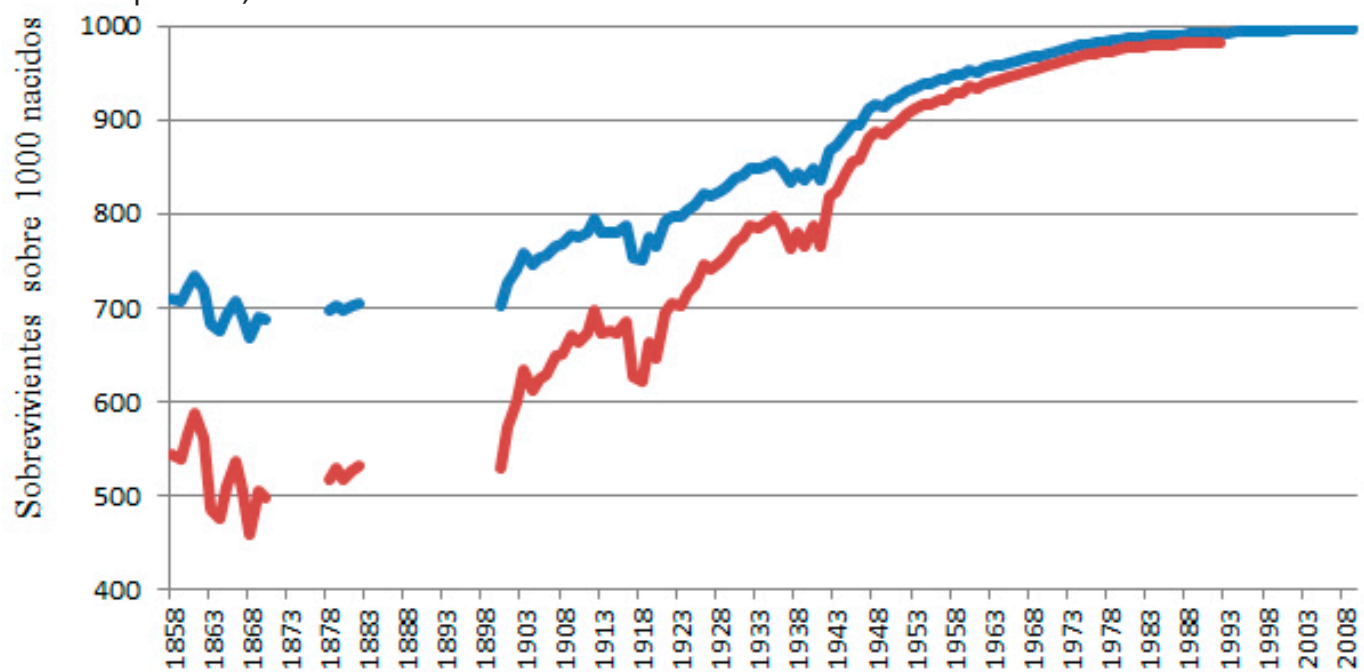

Generaciones, año de nacimiento

\section{Sobrevivientes a los 2 años $\longrightarrow$ Sobrevivientes a los 21 años}

Fuente: Sobrevivientes a los 2 años: elaboración propia a partir de las EMN. Sobrevivientes a los 21 años para las generaciones posteriores a 1910: Blanes 2007 y para las generaciones de 1910 y anteriores: estimación propia a partir de la función ajustada con los datos de Blanes y expresada en la nota 4. La serie de sobrevivientes a los 2 años se refiere a la población de ambos sexos e incluye una estimación de los muertos del primer día para el periodo anterior a 1919. 
mayores de 2 años de manera que todas las generaciones nacidas entre 1900 y 1942 padecieron en algún momento antes de los 21 años, una u otra de aquellas dos crisis de mortalidad o ambas crisis. La serie de sobrevivientes a los 21 refleja como veremos a continuación el fuerte impacto de aquellas dos crisis.

Las dos series del grafico 3 muestran, como era de esperar, las mismas fluctuaciones, reflejando el elevado peso de la mortalidad en los primeros dos años de vida y su importancia relativa sobre la mortalidad antes de los 21 años. No obstante, la distancia entre ambas series se reduce progresivamente, sobre todo a partir de la generación de 1941. Este proceso de convergencia refleja que las generaciones nacidas después de 1941 vivieron en condiciones de salud relativamente mejores a medida que avanzaba su edad. Dicha experiencia no la vivieron siempre las generaciones anteriores del siglo XX porque tuvieron que hacer frente antes de los 21 años a una o dos crisis de mortalidad. La generación de 1900, por ejemplo, padeció una elevada mortalidad en su primera infancia y también en su adolescencia, disminuyendo la proporción de efectivos de aquella generación a $700 \%$ y a $530 \%$ respectivamente a los 2 y 21 años de edad. Las generaciones de 1916 a 1921 padecieron el aumento de la mortalidad infecciosa en su primera infancia e infecciosa y también traumática durante la Guerra Civil y sus proporciones de sobrevivientes a los 2 y 21 años bajaron hasta $750 \%$ y $625 \%$, respectivamente. Las generaciones nacidas a partir de 1942, a diferencia de las anteriores, sí que pudieron conocer mejores condiciones de salud a medida que avanza su edad y por esto la proporción de sobrevivientes a los 21 años de estas generaciones aumentó más rápido que la proporción de sobrevivientes a los 2 años y ambas cifras tendieron a converger.

Con la serie de nacimientos y la serie de la proporción de sobrevivientes a los 21 años de edad, hemos estimado los efectivos absolutos que alcanzaron aqueIla edad de las generaciones masculinas nacidas desde 1858. Esta serie junto con la de los nacimientos están representadas en el gráfico 4. Los espacios vacíos en el siglo XIX se deben a la falta de datos de nacimientos (en muy pocos años) y de las defunciones clasificadas por edades (en un mayor número de años).

La serie de efectivos sobrevivientes a los 21 años muestra, como era de esperar, las fluctuaciones de la natalidad que ya hemos comentado antes. Sin embargo, mientras los nacimientos alcanzaron en muchos años cifras próximas a los 335.000 , los efectivos sobrevivientes a los 21 años no superaron la cifra de 185.000 hasta las primeras generaciones del siglo XX, 285.000 hasta 1957 y la cifra de 335.000 hasta las generaciones últimas del baby boom, nacidas a principios de los años 1970. Nuestro propósito ahora es contrastar esta serie establecida a partir de los datos de las estadísticas del movimiento natural con las series anuales de jóvenes alistados, re-

Gráfico 4. Nacimientos y sobrevivientes a los 21 años de las generaciones masculinas españolas, 1858-2010

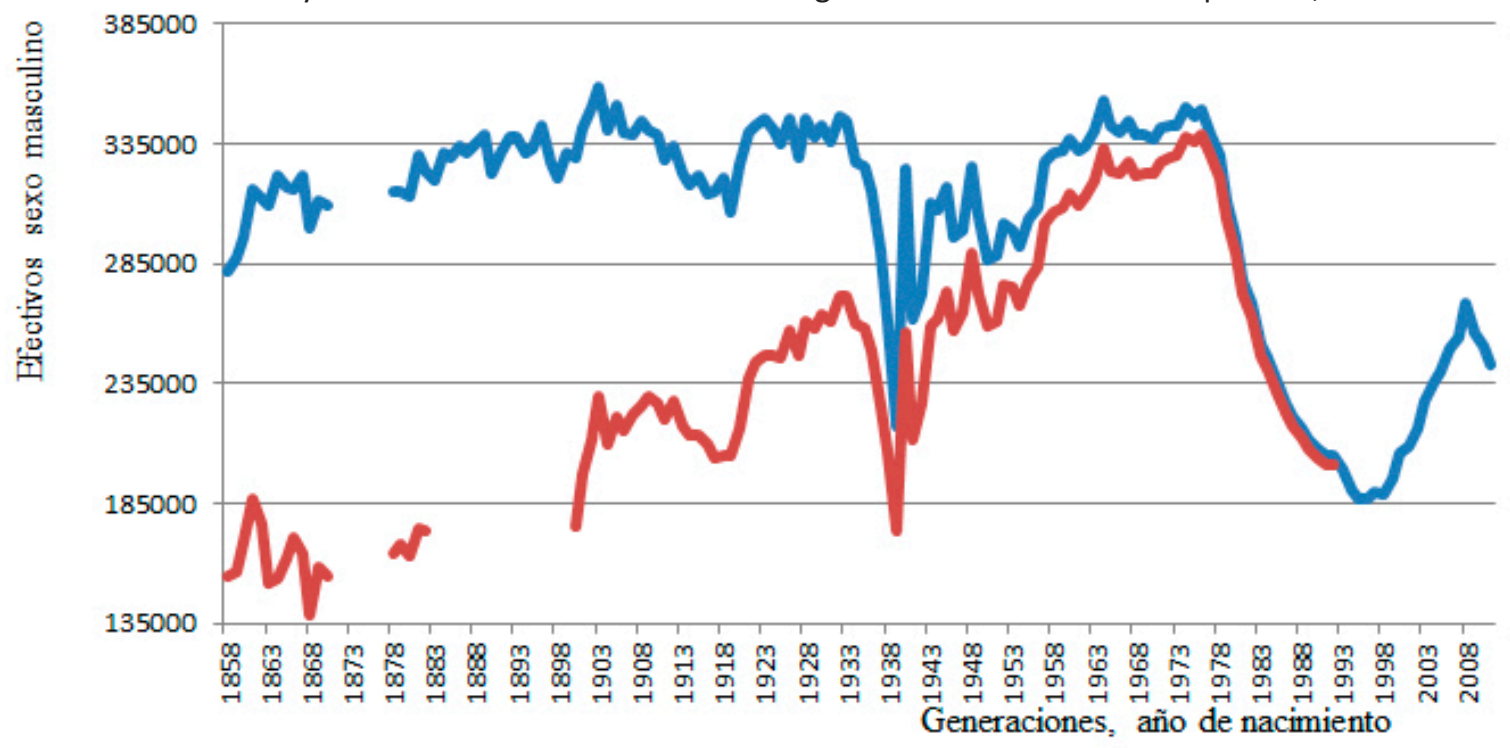

Nacimientos Sobrevivientes a $\operatorname{los} 21$ años

Fuente: elaboración propia a partir de las series de datos de los gráficos 1 y 3 . 
clutados y que formaban el contingente del ejército. De estas tres series de datos del ejército, la más próxima a la nuestra de sobrevivientes a los 21 años es la de los efectivos en el momento del alistamiento y a partir de la cual el ejército establecía las otras dos.

En febrero de cada año los órganos de reclutamiento, ayuntamientos y oficinas consulares, establecían una lista nominal de los mozos que a lo largo del año cumplían la edad requerida para el alistamiento. Esta edad se estableció en 21 años a partir de la generación de 1886 y hasta la de 1966 . Para las generaciones posteriores, de 1967 a 1980, esta edad se avanzó hasta los 19 años, pero entonces la mortalidad era ya muy reducida y las diferencias en los números de efectivos sobrevivientes a los 19 o 21 años eran muy pequeñas. En las generaciones nacidas antes de 1886, los cambios en la edad del alistamiento (20 años para las generaciones de $1837-1866$ y de $1881-85$ y 19 años para las de 1867-80) pudieron afectar en mayor medida al número de efectivos, porque la mortalidad era entonces mucho más elevada. Pero la serie de alistados que hemos reconstruido a partir de los anuarios estadísticos militares y de los anuarios estadísticos del INE, empieza en la generación de 1879 y hemos preferido no modificar la edad de referencia de 21 años de la serie de sobrevivientes, hasta disponer de más datos demográficos de las generaciones más antiguas y determinar mejor otros factores que pudieran afectar la cobertura del alistamiento ${ }^{11}$.
El gráfico 5 compara la serie de efectivos sobrevivientes a los 21 años (del gráfico 4) con la serie de efectivos alistados y como esta última empieza más tarde para completarla hemos añadido al gráfico una tercera serie más larga: la de los efectivos al reemplazo, referida como las otras dos al año de nacimiento de cada generación. Esta tercera serie comprende a los alistados de cada generación pero comprende una parte variable de los alistados de años anteriores. Esta parte era relativamente pequeña excepto en algunos años, por ejemplo en años de guerra o expectativas de conflicto cuando se extendía el reclutamiento mediante el recurso de incorporar a efectivos de alistamientos anteriores. Las diferencias entre efectivos del reemplazo y del alistamiento son máximas por ejemplo, en las generaciones nacidas a finales de los años 1870, que fueron movilizadas en 1898. La concordancia entre las series de sobrevivientes y la de alistados, que empieza con la generación de 1878 , es bastante significativa, lo que indica que el alistamiento era también bastante universal entonces.

Para el siglo XIX, son más largas las series de estadísticas militares que las de sobrevivientes establecidas a partir de las estadísticas más tardías e interrumpidas del movimiento natural de la población. En cambio en el siglo XX, mientras se dispone de estas últimas de forma regular, existe un vacío importante en la publicación de las estadísticas militares, que se alarga de 1936 a 1953 y corresponde a las generaciones de 1915 a 1932. En los años

Gráfico 5. Efectivos sobrevivientes a los 21 años, al alistamiento y al reemplazo de las generaciones masculinas españolas, 1837-1954

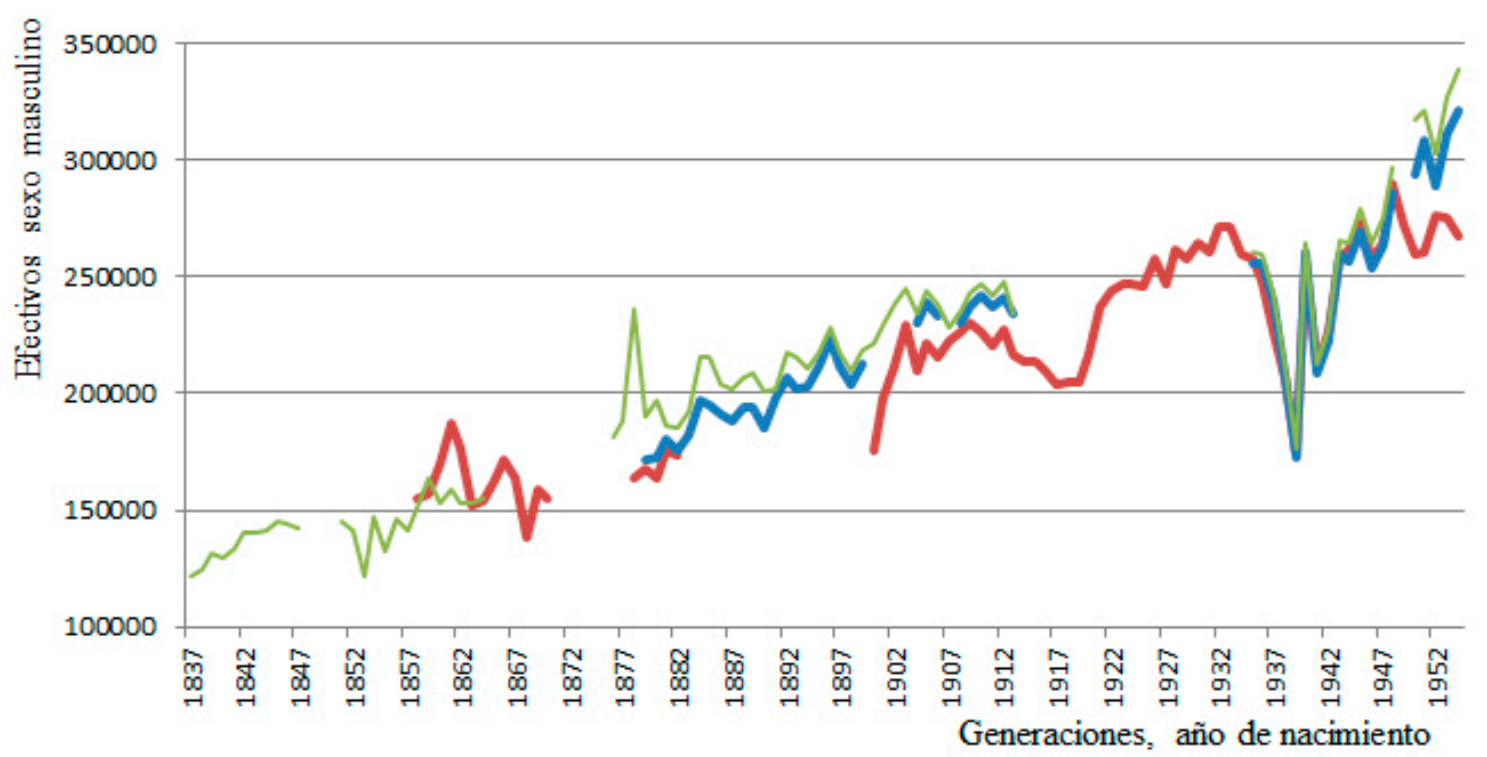

- Sobrevivientes a $\operatorname{los} 21$ años Efectivos alistados Efectivos del reemplazo

Fuente: serie de sobrevivientes del gráfico 4 y series de efectivos alistados y del reemplazo: Reseña Estadística de 1888, Anuarios Estadisticos de España anteriores a 1937 del INE, Estadísticas de Reclutamiento y Reemplazo de 1912-14, 1915-17, 1918-20 del Instituto Geográfico y Estadístico y Anuario Estadístico Militar de 1954 del Ministerio de Defensa. 
que se pueden cubrir con ambas series, las concordancias en los niveles y las fluctuaciones anuales de ambas son muy significativas, lo que demuestra que la cobertura del alistamiento era muy satisfactoria. El hecho de que el número de alistados sobrepase de manera significativa a los sobrevivientes de algunas generaciones -las nacidas alrededor de 1910 y de 1950- podría deberse a que los alistamientos registraban hijos de españoles que habían nacido en el extranjero, y sobre todo en los años que se produjeron más retornos de emigrantes debido a las crisis económicas de las décadas de 1930 y 1970, que correspondían a alistados de las generaciones nacidas en torno a 1910 y 1950 respectivamente. Sin embargo la discrepancia en este último periodo de la serie también se debe a que se avanzó entonces la edad de alistamiento y se distribuyeron los efectivos de una generación entre varios reemplazos. En este caso este factor de discrepancia podrá corregirse cuando dispongamos de más datos.

Es significativo que la serie de alistamiento de las dos últimas décadas del siglo XIX ajuste bastante bien con las estimaciones de sobrevivientes de las generaciones nacidas hacia 1880 , de forma que la serie de alistamiento de 1880 a 1900 sea muy interesante para reconstruir la evolución de la mortalidad de niños y jóvenes durante aquellos años. Así mismo, cabe destacar que el servicio militar no fue efectivamente obligatorio para las generaciones anteriores a 1891, alistadas antes de 1912, por lo que se suponía que la cobertura del alistamiento pudiera ser para las generaciones anteriores muy incompleta. Ciertamente las generaciones anteriores a 1891 podían evitar el servicio militar mediante la redención en metálico o la contratación de un substituto y escoger esta opción antes del alistamiento. La cobertura del alistamiento para las generaciones nacidas alrededor de 1880 nos parece comparada con las estimaciones de sobrevivientes muy buena, lo que indicaría que los que se aprovechaban de esta posibilidad de evitar el servicio militar lo hacían generalmente después del alistamiento y probablemente después de saber si iban a formar parte o no del reemplazo y con posterioridad a conocer su destino. Desde la generación de 1891 el alistamiento era obligatorio y universal y los mozos que disponían de recursos suficientes y otras características, como un determinado nivel de educación, vieron sus ventajas limitadas a la posibilidad de reducir su tiempo de servicio.

Una vez realizado el alistamiento, los mozos alistados debían presentarse para ser examinados y clasificados en las cuatro grandes categorías siguientes: útiles; excluidos de forma definitiva o temporal por motivos de salud o insuficiente desarrollo físico; exceptuados por determinadas situaciones familiares y por ejercer determinadas actividades; y finalmente, los que no se presentaban para ser examinados y eran declarados prófugos.

Al primer grupo de mozos útiles, se añadía un número generalmente pequeño de alistados de años anteriores y la suma de ambos constituía el contingente anual de mozos que se incorporaban al servicio militar. El contingente más los excluidos, los exceptuados y los prófugos sumaban el total de los efectivos del reemplazo. El gráfico 6, muestra la evolución de las proporciones que representaron estos cuatro grupos sobre los reemplazos del periodo 1895-1933.

Gráfico 6. Distribución (en \%) de los efectivos de los reemplazos de 1895 a 1933 en tres categorías: contingente, excluidos y exceptuados y prófugos

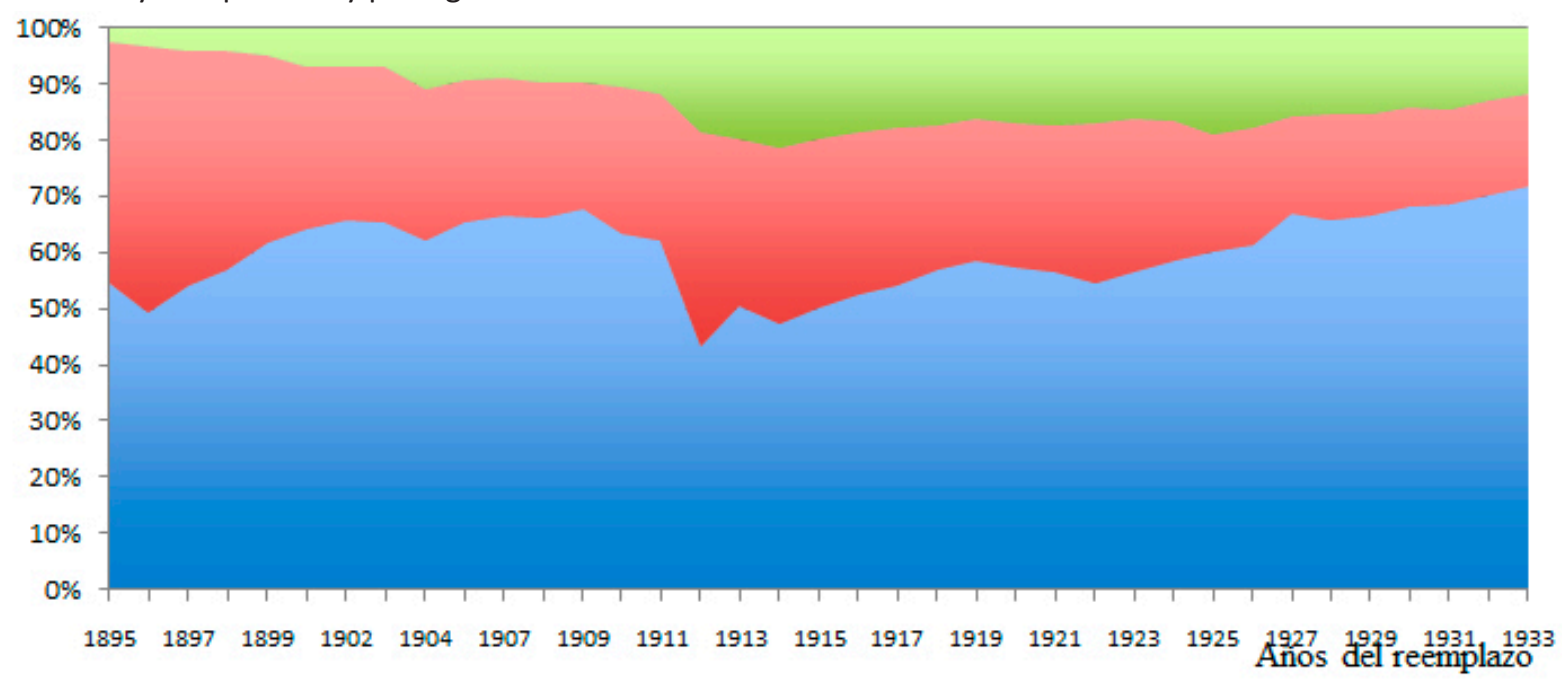

- efectivos del contingente = efectivos excluidos y exceptuados efectivos prófugos

Fuente: a partir de las fuentes del gráfico 5 . 
La proporción de excluidos por motivos de salud y desarrollo físico cambió a veces de forma brusca debido a alteraciones en los criterios de clasificación: en 1912 por ejemplo, se aplicaron criterios más restrictivos para identificar a los mozos útiles en función de su desarrollo físico y estado de salud y en 1914 y 1924 estos criterios se relajaron. No es extraño por tanto que a partir de aquellos años se observen primero un aumento y después dos descensos respectivamente en la proporción de mozos excluidos por razones de salud. La proporción de prófugos por el contrario siguió una evolución más regular. Aumentó progresivamente del $3 \%$ hasta el $22 \%$ entre 1895 y 1914 , y tendió a disminuir posteriormente y sobre todo entre 1929 y 1933, hasta alcanzar un $12 \%$ del reemplazo en este último año. La elevada emigración exterior de finales del siglo XIX y principios del XX facilitó el incremento de la proporción de prófugos, de la misma forma que la fuerte reducción del saldo migratorio exterior en los años 1920 y primeros 1930 contribuyó entonces a su disminución.

\section{MORBILIDAD Y MORTALIDAD EN LAS ESTADÍSTICAS SANITARIAS MILITARES}

Estimar la morbilidad de las generaciones del pasado es mucho más difícil que estimar su mortalidad. Las fuentes antropométricas, que informan por ejemplo de la estatura, pueden proporcionar indicadores indirectos del impacto de la enfermedad en el crecimiento de los niños y de los adolescentes ${ }^{12}$. Otras fuentes que han utilizado los historiadores para evaluar la morbilidad son las estadísticas de las compañías de seguros, de sociedades de ayuda mutua, etc. Dos de las principales limitaciones de estas fuentes son su representatividad y la ausencia muchas veces de datos sobre la edad de los afiliados lo que no permite estimar la morbilidad en función de la edad. Este segundo problema no lo presentan las estadísticas de morbilidad de los soldados - un colectivo de edad uniforme- y el primer problema es mucho menor en este caso ya que éstas cubren un colectivo de la población, aunque solo la masculina, muy amplio aunque como veremos a continuación no la totalidad de los jóvenes en edad militar. Las observaciones de mortalidad y morbilidad que hemos reunido se establecieron sobre los efectivos del contingente, y la proporción que éstos representaban sobre el total de efectivos del reemplazo, osciló entre el $50 \%$ y el $70 \%$ a lo largo del periodo analizado. Las variaciones en la proporción de exceptuados sólo son importantes entre 1895 y 1897 y no podemos precisar qué efectos pudieron tener sobre los indicadores de salud del contingente. Los cambios en la proporción de excluidos por razones de desarrollo físico y salud es previsible que afectaran aquellos indicadores. De la misma forma que el incremento de las proporciones de excluidos en 1912 pudo contribuir a mejorar las condiciones de salud del contingente, su reducción a partir de entonces y sobre todo a partir de 1924 pudo por el contrario tener un impacto negativo. No se puede sin embargo olvidar que la paulatina reducción de la proporción de excluidos refleja también una mejora de las condiciones generales de salud de la población masculina. Finalmente, las variaciones en la proporción de prófugos creemos que pueden producir un sesgo en nuestros indicadores de salud por el motivo que argumentamos a continuación. No presentarse al examen y clasificación después del alistamiento era un comportamiento perseguido y penalizado y por esto cabe suponer que la gran mayoría de los mozos que no se presentaron es porque tenían elevadas probabilidades de no pertenecer a ninguna de las categorías que les hubieran permitido ser excluidos o exceptuados del servicio militar. Por esta razón creemos que la elevada proporción de prófugos, que se registró entre el $15 \%$ y el $22 \%$ en los reemplazos de 1912 a 1929, sesgó, en un sentido negativo, las observaciones sobre el estado de salud de los alistados que establecieron los militares, lo que puede explicar, junto con el cambio de criterio de clasificación al que hemos aludido antes, el aumento de la proporción de excluidos a partir de 1912 y una parte también del aumento de la morbilidad y mortalidad de los efectivos que cumplieron el servicio militar en los años siguientes que examinaremos a continuación.

La estadística sanitaria del ejército, como se ha explicado al principio del artículo, proporciona datos de los soldados en filas muertos y hospitalizados desde mediados del siglo XIX. Para estimar la mortalidad y morbilidad de este colectivo, los datos de muertos y hospitalizados se refirieron a partir de la década de 1880 al promedio anual del efectivo de soldados en filas. En el Apéndice 1 se presenta una tabla con los datos disponibles de morbilidad, mortalidad y días de hospitalización por enfermo para los años comprendidos entre 1880 y 1933. La construcción de la serie se ha realizado, en primer lugar, a partir del seguimiento de las series estadísticas sanitarias militares disponibles $^{13}$ y de los anuarios estadísticos de España ${ }^{14}$, y después a través de la contrastación y complementación, cuando ha sido necesario, con los proporcionados por diversos artículos aparecidos en las revistas de sanidad militar publicadas durante esos años ${ }^{15}$. 
El gráfico 7 muestra la evolución del promedio anual de soldados que cumplían el servicio militar en la península e islas adyacentes de 1886 a 1933. Junto a esta serie se ha representado la serie anual de los efectivos del contingente, que hemos podido reconstruir solo a partir de 1895. Para facilitar la comparación, la serie de los efectivos del contingente la hemos desplazado un año, ya que el contingente de 1895 cumple el primer año completo de servicio militar en 1896. Las diferencias entre ambas series se explican porque no todos los efectivos del contingente se incorporaban a filas y porque la duración media del servicio militar no era igual a un año. La principal discrepancia entre ambas series que se produce a partir de 1912 se debe fundamentalmente al aumento primero, y a la posterior reducción después de la duración media del servicio militar. En los primeros años de la década de 1920 los efectivos en filas alcanzaron un valor máximo de 250.000 , mientras que antes de 1912, la mayor parte de los años no alcanzaron la cifra de los 100.000. La estimación realizada por el ejército de los efectivos en filas es imprescindible para estimar la mortalidad y la morbilidad de aquel colectivo.

El gráfico 8 muestra la evolución de la tasa de mortalidad de los soldados en filas causada por todas las enfermedades, excepto las calificadas como externas (traumatismos, homicidios y suicidios), que normalmente eran más elevadas entre los soldados que entre los civiles. Esta tasa de mortalidad de los soldados puede compararse para los mismos años con la tasa bruta de mortalidad de la población española y, a partir de 1911, con la tasa específica de mortalidad por todas las causas de los hombres españoles de 20-24 años, calculada anualmente por Blanes en su tesis doctoral. Aunque la selección de los soldados se establecía en gran medida en función de su estado de salud y nuestro indicador no comprende las muertes por causas externas, la mortalidad de los soldados no fue, en muchos de los años contemplados, significativamente inferior a la mortalidad del conjunto de la población masculina de 20-24 años. Además, otra diferencia que se observa entre ambos colectivos es la mayor fluctuación de las tasas de mortalidad de los soldados en filas, que sugiere que el peso relativo de las enfermedades infecciosas en este colectivo era mayor debido probablemente a sus particulares condiciones de vida y concretamente, a su mayor densidad habitacional y hacinamiento con respecto al resto de la población ${ }^{16}$. Gracias de nuevo a la tesis de Blanes, disponemos de la tasa de mortalidad por enfermedades infecciosas de la población masculina de 20-24 años, que muestra la misma tendencia que la tasa de mortalidad general pero el aumento del peso relativo de la mortalidad infecciosa en 1918 . En este año la tasa de mortalidad de los soldados fue inferior y no olvidemos que esta tasa contiene las enfermedades infecciosas y no infecciosas, lo que podría deberse al efecto de selección por el criterio de mejor salud de los soldados, y que durante aquel año se rechazará la incorporación a filas de los infectados por gripe. No podemos descartar, por ahora, otras razones como

Gráfico 7. Evolución del promedio anual de los efectivos en filas y del contingente, España 1886-1933

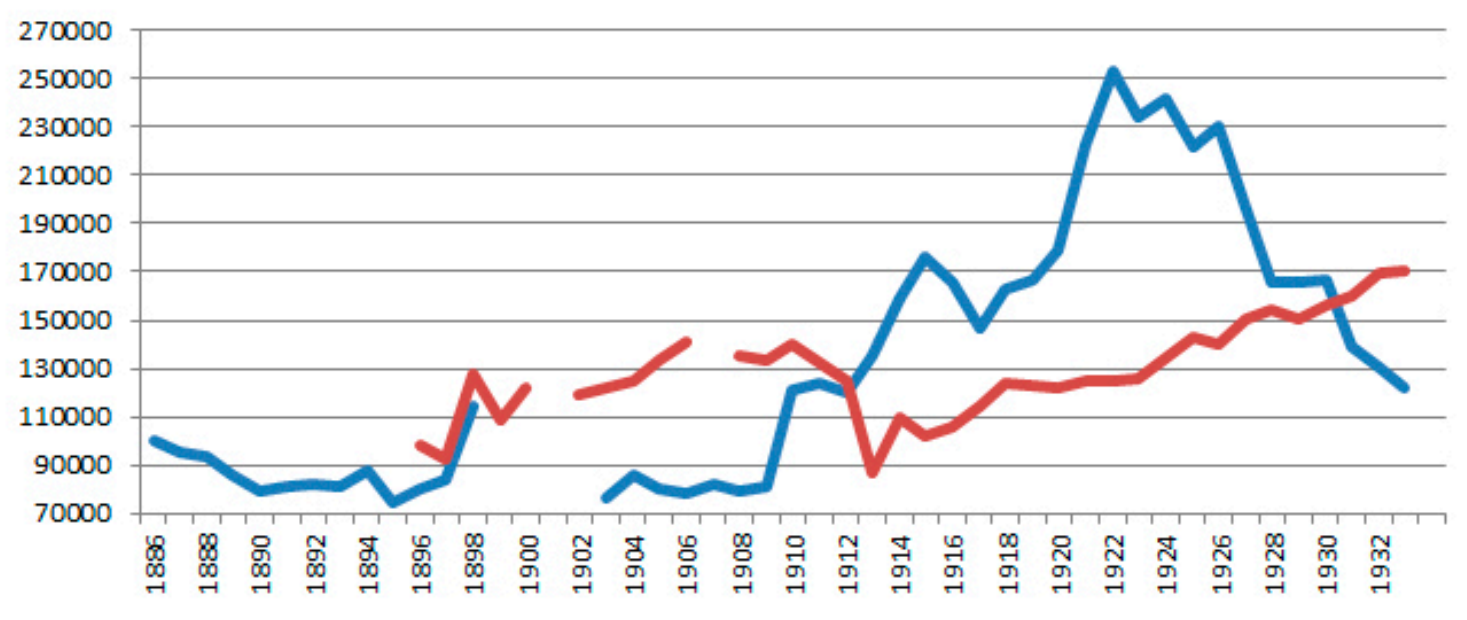

"prom edio anual de efectivos en filas

efectivos del contingente del año anterior

Fuente: a partir de la fuentes del gráfico 5 . 
una mayor capacidad de los servicios sanitarios del ejército para hacer frente a la epidemia de gripe o un ocultamiento de los efectos de ésta sobre el contingente. No obstante, antes y después de 1918 son muy frecuentes y acusadas las fluctuaciones de la mortalidad lo que nos conduce a pensar que se debieron a causas infecciosas que afectaban en mayor medida a la población hacinada en los cuarteles. Las exploraciones que hemos hecho sobre las enfermedades que explicarían aquellos picos de la sobremortalidad de los soldados señalan que la tuberculosis tuvo el mayor protagonismo durante todo el periodo, si bien empezó a disminuir paulatinamente desde el cambio de siglo, aunque con puntas ocasionales; la viruela mantuvo una incidencia destacada hasta finales de la década de 1880; la fiebre tifoidea, el paludismo y varias enfermedades respiratorias (bronquitis y neumonías) seguirían en importancia.

Dadas las características de la vida en los cuarteles, las estadísticas hospitalarias militares tienen una cobertura de los efectivos enfermos, sus causas y la duración de sus dolencias que no disponemos para la población civil. Para la misma población de soldados en filas la estadística del ejército proporciona el número anual de soldados hospitalizados y la duración media de su estancia en el hospital, haciendo factible calcular, gracias a que conocemos el efectivo de soldados, el número medio de días al año que pasaba hospitalizado un soldado. El gráfico 9 muestra para el periodo 1886-1933 la evolución de este indicador y de la tasa de mortalidad representada en el gráfico anterior. Las fluctuaciones anuales de ambos indicadores coinciden significativamente, pero mientras el número anual de días de hospitalización por soldado se redujo de 12 a 9 días entre 1886 y 1912, la frecuencia de defunciones se redujo mucho más, de 13 a 3 muertos al año (sobre 1000 soldados) en el mismo periodo. En los años siguientes, entre 1912 y 1927, se produjo un aumento de la morbilidad que alcanzó niveles muy parecidos a los de los peores años del siglo XIX. La mortalidad también aumento en estos años pero no alcanzo sin embargo, incluso en 1918, los niveles de los años más críticos del siglo XIX. En definitiva, lo que señalan estas series es que la morbilidad no siguió una evolución paralela a la de la mortalidad, y que por lo tanto no debiera utilizarse el indicador de una de estas variables en substitución del otro o inferir la evolución de una de las dos variables a partir de la otra.

Gráfico 8. Tasa de mortalidad por enfermedades no externas de los soldados en filas, comparada con las tasas indicadas de la población española, 1886-1933

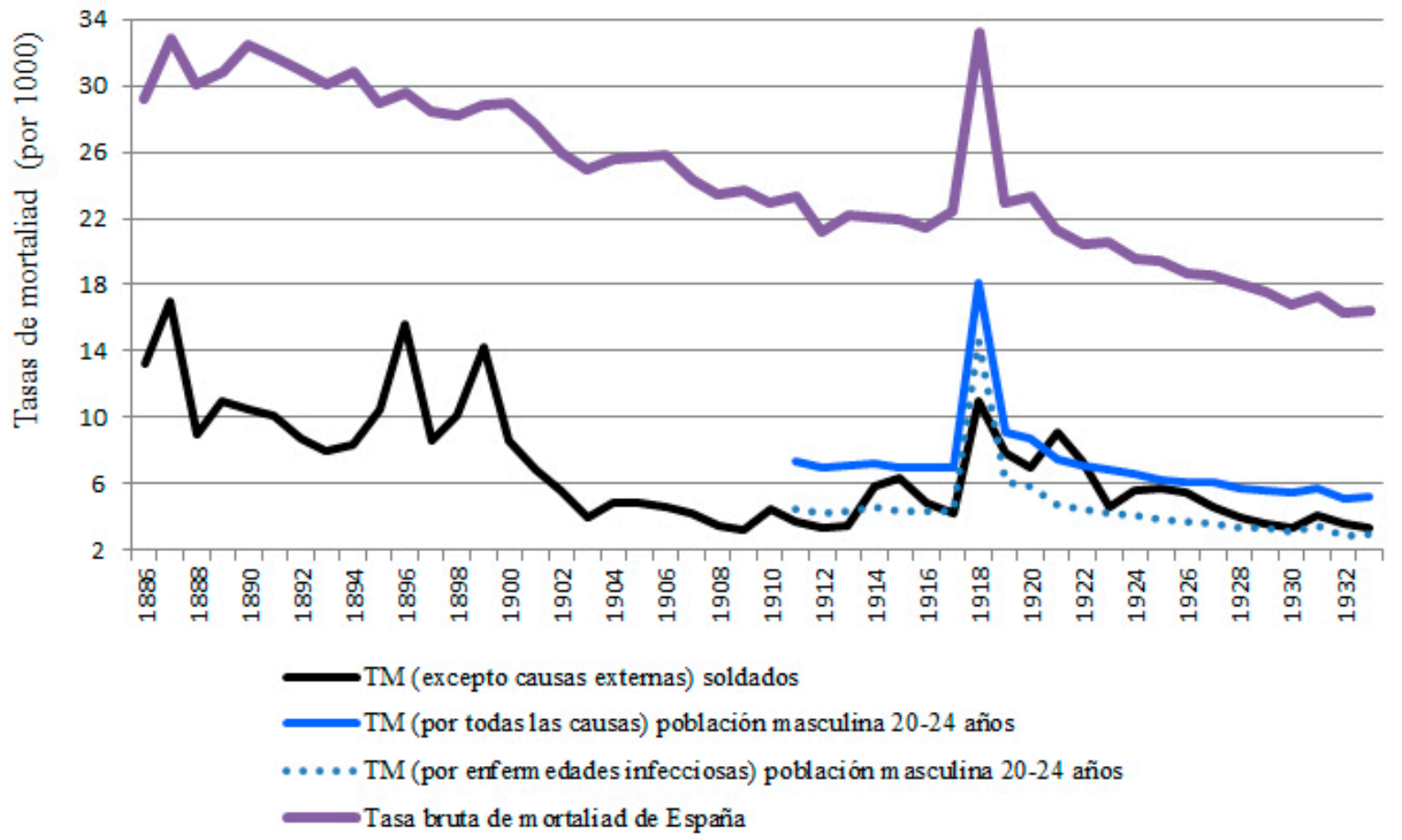

Fuente: la TBM de España: Nicolau 2005, pp.124-125 ; las tasas de mortalidad de la población masculina de 20-24 años: Blanes 2007; la de los soldados elaboración propia a partir de las fuentes del gráfico 5 y del Apéndice 1. 
Gráfico 9. Evolución de la prevalencia de la morbilidad y la mortalidad de los soldados en filas, 1886-1933

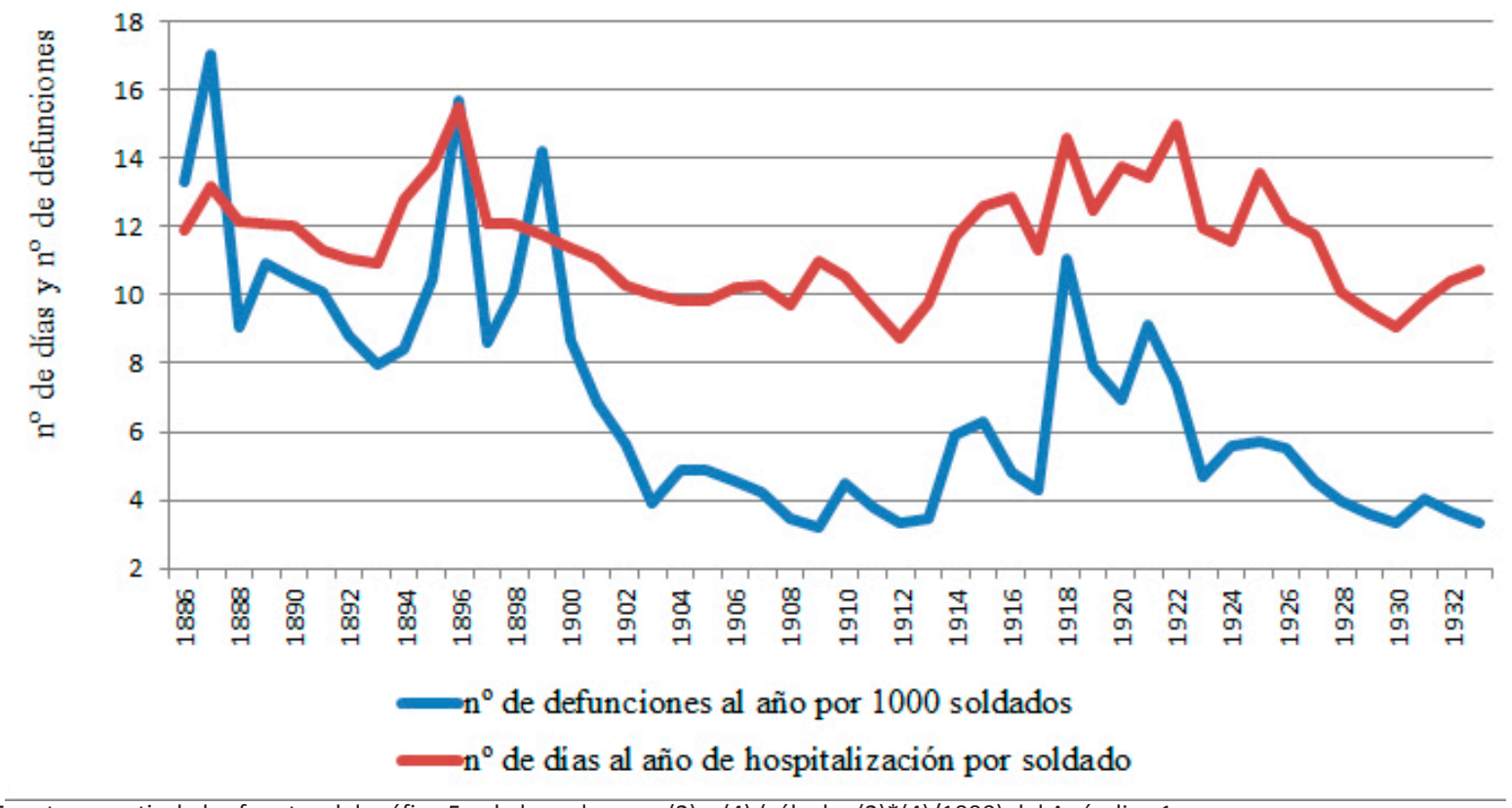

Fuente: a partir de las fuentes del gráfico 5 y de las columnas (2) y (4) (cálculo: (2)*(4)/1000) del Apéndice 1.

Existen bastantes evidencias de otras poblaciones y países que muestran que la morbilidad y la mortalidad no siguen evoluciones paralelas, y que muestran que la morbilidad aumentó durante el descenso o transición de la mortalidad (ver por ejemplo Riley, 1990 y 1989). La explicación de este hecho es compleja y controvertida (ver por ejemplo Johansson, 1991; Harris, 1999 y Floud y otros, 2011) y una de las razones es porque la definición y delimitación de la enfermedad es mucho más imprecisa y variable que la de la mortalidad lo que dificulta su estimación y finalmente su interpretación. Una explicación por ejemplo que se ha dado del aumento de la prevalencia de la morbilidad, es el aumento de la proporción de los efectivos de la población más vulnerables y con afecciones crónicas que se habría producido con el descenso de la mortalidad y la ampliación de las probabilidades de sobrevivencia. Otra explicación, muy plausible también, es que el descenso de la mortalidad y los factores que lo producen aumentan la percepción de la enfermedad y la atención a los enfermos, lo que en definitiva elevaría también la prevalencia de la morbilidad. Estas dos argumentaciones no son excluyentes pero el aumento de la morbilidad es interpretado de forma mucho más optimista en la segunda explicación que en la primera. En la segunda, el aumento sostenido de los indicadores de morbilidad reflejaría una mayor prevención sanitaria y atención a los enfermos, mientras que en la primera explicación aquel aumento reflejaría una mayor carga de la enfermedad entre la población y en definitiva que una parte significativa de los años ganados de vida o del aumento de la esperanza de vida se sacrifican con una mayor prevalencia de la enfermedad.

\section{CONCLUSIONES}

Esperamos poder prolongar las series que hemos reconstruido en este trabajo, para unos años anteriores del siglo XIX y varias décadas del siglo XX, para responder a las preguntas de la presentación. Creemos que una perspectiva temporal más larga nos permitiría distinguir si el aumento de la morbilidad que hemos observado durante la segunda y tercera décadas del siglo XX fue un hecho excepcional y acotado en el tiempo, o una constante del proceso de la transición y descenso de la mortalidad. Las evidencias de otros países, como hemos visto, coinciden y señalan la segunda posibilidad: que la prevalencia de la morbilidad aumentó durante el descenso secular de la mortalidad. Independientemente de cual sea la respuesta a la pregunta anterior, las series que hemos presentado en este trabajo señalan dos factores a considerar también en el aumento de la morbilidad de los soldados españoles durante los años siguientes a la implantación del servicio militar obligatorio en 1912: el incremento del promedio anual de los efectivos en filas, de 120.000 en 1912 a más de 250.000 en 1922; 
y, la reducción entre los mismos años de la proporción de excluidos por motivos de salud (ver gráficos 7 y 8). Los responsables de la sanidad militar aludieron frecuentemente al segundo factor, al que atribuyeron aquel aumento en la morbilidad, no consideraron sin embargo el primer factor - el fuerte incremento del número de soldados - y sus posibles consecuencias en las condiciones sanitarias de los cuarteles y en la difusión y prevalencia de la morbilidad infecciosa.

La preocupación y perspectiva de análisis de los responsables de la sanidad militar española estuvo muy focalizada en las variaciones de la morbilidad y la mortalidad en el muy corto plazo y una prueba de ello es que entre sus publicaciones no hemos encontrado ningún trabajo que reconstruya las series temporales de los indicadores de aquellas variables y que realice un análisis comparativo de su evolución. Uno de los motivos que, en nuestra opinión y durante el periodo analizado en esta comunicación, justificaría esta falta de perspectiva en sus análisis, es la importancia relativa que tenían las enfermedades infecciosas entre la tropa, que causaban importantes fluctuaciones en su mortalidad y que ocultaban demasiado a menudo la tendencia de fondo y el descenso de la mortalidad que se estaba produciendo desde las últimas décadas del siglo XIX. Las series de los indicadores de mortalidad de los soldados y de la población española que hemos reunido en los gráficos 3 y 8 muestran que la transición de la mortalidad se interrumpió durante una secuencia de varios años, anteriores y posteriores a la epidemia de gripe de 1918, por lo que la explicación del aumento de la morbilidad y la mortalidad de los soldados en aquel periodo debe enmarcarse también en la evolución de las condiciones de salud del conjunto de la población española. Creemos en definitiva que para conocer mejor las relaciones entre la morbilidad y la mortalidad es necesario disponer de una perspectiva temporal larga y a la vez mantener en la medida de lo posible, como hemos intentado hacer en este trabajo, una perspectiva comparativa que muestra las particularidades y similitudes de la evolución de la salud de los soldados y del resto de la población. Ambas cosas no pudieron hacerlas los responsables de elaborar las estadísticas de sanidad militar pero lo que sí hicieron y creemos que bastante bien es recoger la información necesaria para realizar aquella tarea. La realización de este trabajo nos ha convencido de que existe aún una importante riqueza por explotar en las estadísticas sanitarias del ejército español. Estas estadísticas pueden contrastarse con las procedentes de otras fuentes, como las estadísticas del movimiento natural, los censos y las sanitarias. $Y$ cuando estas últimas fuentes no existen como sucede en gran parte del siglo XIX o son muy incompletas, como en el caso de las estadísticas de morbilidad de la población, las fuentes militares ofrecen al menos observaciones regulares de un sector concreto de la población. Un sector limitado, claro está, del sexo masculino y de un grupo específico de edad, pero que fue observado de forma sistemática, a lo largo de un lapso de tiempo muy prolongado. 


\section{APÉNDICE I}

Tabla de morbilidad y mortalidad del Ejército español (1880-1933)

(Península, Baleares y Canarias)

(Exceptuando muertos y heridos en combate, en accidentes y en suicidios)

\begin{tabular}{ccccc}
\hline Año & $\begin{array}{c}\text { Efectivo medio } \\
\text { en filas }\end{array}$ & $\begin{array}{c}\text { Morbilidad } \\
\text { (soldados } \\
\text { hospitalizados \%) }\end{array}$ & $\begin{array}{c}\text { Mortalidad } \\
\text { (soldados muertos \%o) }\end{array}$ & $\begin{array}{c}\text { Número medio de días de } \\
\text { hospitalización por } \\
\text { enfermo }\end{array}$ \\
\hline
\end{tabular}

(1) (2) (3) (4)

\begin{tabular}{|c|c|c|c|c|}
\hline 1880 & 88269 & 478 & 13,50 & \\
\hline 1881 & 82503 & 560 & 15,70 & \\
\hline 1882 & 93820 & 598 & 18,90 & 24 \\
\hline 1883 & 95886 & 542 & 18,60 & \\
\hline 1884 & 94595 & 580 & 16,70 & \\
\hline \multicolumn{5}{|l|}{1885} \\
\hline 1886 & 100434 & 496 & 13,49 & 24 \\
\hline 1887 & 95284 & 507 & 16,00 & 24 \\
\hline 1888 & 93274 & 466 & 9,02 & 26 \\
\hline 1889 & 86100 & 503 & 10,92 & 24 \\
\hline 1890 & 79387 & 500 & 10,44 & 24 \\
\hline 1891 & 80968 & 453 & 10,06 & 25 \\
\hline 1892 & 82014 & 424 & 8,80 & 26 \\
\hline 1893 & 81268 & 438 & 7,98 & 25 \\
\hline 1894 & 87585 & 491 & 8,61 & 26 \\
\hline 1895 & 73961 & 528 & 10,47 & 26 \\
\hline 1896 & 80181 & 596 & 15,67 & 26 \\
\hline 1897 & 83737 & 484 & 8,63 & 25 \\
\hline 1898 & 114120 & 526 & 10,14 & 23 \\
\hline 1899 & & 490 & 14,21 & 24 \\
\hline 1900 & & 407 & 8,64 & 28 \\
\hline 1901 & & 426 & 6,89 & 26 \\
\hline 1902 & & 411 & 5,65 & 25 \\
\hline 1903 & 76253 & 371 & 3,93 & 27 \\
\hline 1904 & 85770 & 379 & 4,87 & 26 \\
\hline 1905 & 79768 & 351 & 4,85 & 28 \\
\hline
\end{tabular}




\begin{tabular}{|c|c|c|c|c|}
\hline Año & $\begin{array}{l}\text { Efectivo medio } \\
\text { en filas }\end{array}$ & $\begin{array}{c}\text { Morbilidad } \\
\text { (soldados } \\
\text { hospitalizados \%o) }\end{array}$ & $\begin{array}{c}\text { Mortalidad } \\
\text { (soldados muertos \%o) }\end{array}$ & $\begin{array}{l}\text { Número medio de días de } \\
\text { hospitalización por } \\
\text { enfermo }\end{array}$ \\
\hline 1906 & 78538 & 394 & 4,57 & 26 \\
\hline 1907 & 81655 & 410 & 4,24 & 25 \\
\hline 1908 & 79503 & 387 & 3,64 & 25 \\
\hline 1909 & 81321 & 439 & 3,18 & 25 \\
\hline 1910 & 121076 & 438 & 4,48 & 24 \\
\hline 1911 & 123668 & 399 & 3,60 & 24 \\
\hline 1912 & 120391 & 349 & 3,32 & 25 \\
\hline 1913 & 134978 & 408 & 3,43 & 24 \\
\hline 1914 & 158645 & 509 & 5,90 & 22 \\
\hline 1915 & 176295 & 547 & 6,31 & 23 \\
\hline 1916 & 166035 & 514 & 4,81 & 25 \\
\hline 1917 & 147130 & 472 & 4,26 & 24 \\
\hline 1918 & 163082 & 634 & 11,03 & 23 \\
\hline 1919 & 166651 & 567 & 7,47 & 22 \\
\hline 1920 & 178570 & 598 & 6,95 & 23 \\
\hline 1921 & & 706 & 9,15 & 19 \\
\hline 1922 & 253523 & 652 & 7,37 & 23 \\
\hline 1923 & 234053 & 497 & 4,65 & 24 \\
\hline 1924 & & 610 & 5,55 & 19 \\
\hline 1925 & & 616 & 5,70 & 22 \\
\hline 1926 & 230733 & 643 & 5,49 & 19 \\
\hline 1927 & & 619 & 4,57 & 19 \\
\hline 1928 & 165891 & 504 & 3,97 & 20 \\
\hline 1929 & 165672 & 396 & 3,61 & 24 \\
\hline 1930 & 166281 & 394 & 3,31 & 23 \\
\hline 1931 & 138761 & 447 & 4,07 & 22 \\
\hline 1932 & 130302 & 453 & 3,65 & 23 \\
\hline 1933 & 121650 & 512 & 3,34 & 21 \\
\hline
\end{tabular}

Fuentes: las citadas en las notas no 10,11 y 12 


\section{NOTAS}

1. La investigación se ha realizado en el marco de los proyectos Población, alimentación y niveles de vida, España siglos XIX y XX, HAR2013-47182-C02-1-P y Dynamic Multidimensional Well-Being Assessment, 2014 SGR 591. Agradecemos a los evaluadores anónimos los comentarios y sugerencias que han hecho a nuestro trabajo, y que han contribuido a mejorar el contenido del presente artículo.

2. Instituto Nacional de Estadística, Anuario Estadístico de España 1862-1865, pp. 121-134 y Anuario Estadístico de España 1866-1867, pp. 286-307.

3. Por ejemplo, Anónimo (1887), "Cuadros estadísticos referentes a la asistencia prestada en los hospitales y enfermerías militares durante el año 1886", Revista de Sanidad Militar, I (12), pp. 197-198.

4. Por ejemplo, las Memorias-Resúmenes de la Estadística Sanitaria del Ejército no ofrecen la cifra absoluta de muertos, sino la proporcional, mientras que en algunos años los Anuarios Estadísticos de España sí facilitan ese dato.

5. Instituto Nacional de Estadística, Anuario Estadístico de España 1862-1865, pp. 126-127.

6. Desconocemos por que razón las estadísticas sanitarias militares no siguieron la clasificación de enfermedades de la estadísticas sanitarias civiles, pero así fue al menos hasta 1936, Sánchez Ocaña $(1992,59)$

7. Sobre las dificultades de identificación y ordenación de enfermedades en series históricas es imprescindible el estudio y las propuestas de homogeneización en Bartomeu-Mestre (2003).

8. En las series más antiguas no se diferenciaba entre tifus y fiebre tifoidea; es desde principios del siglo XX cuando empieza la distinción entre "tifus abdominal" (fiebre tifoidea) y tifus exantemático.

9. Las biografías de estas publicaciones son consultables en la Hemeroteca Digital de la Biblioteca Nacional de España: http://hemerotecadigital.bne.es/index.vm

10. Además véanse las series anuales de Italia en http://www. mortality.org

11. La legislación sobre el reclutamiento fue abundante y compleja, dada la reiteración de modificaciones sucesivas a lo largo de estos años. Las fuentes primarias son: Ministerio de la Guerra, Novísima ley de reemplazos de 10 de Enero de 1877, publicada en la Gaceta del mismo mes, reformando las de 30 de Enero de 1856, 29 de Marzo de 1870 y 17 de Febrero de 1873; Ley de Reclutamiento y Reemplazo del Ejército de 11 de Julio de 1885; Ley de Reclutamiento y Reemplazo del Ejército de 11 de Julio de 1885, modificada por la de 21 de Agosto de 1896; Ley de Reclutamiento y Reemplazo del Ejército de 27 de Febrero de 1912, conforme a la Ley de Bases del 29 de Junio de 1911.
12. En España son numerosos y de gran calidad los estudios antropométricos, sobre todo sobre la estatura como indicador de la evolución intergeneracional de la salud y el bienestar biológico: (Gómez-Mendoza y Pérez-Moreda, 1995; Martínez-Carrión, 2014; Quiroga Valle, 2001, Spijker, Jeroen, Cámara, Antonio D. y Blanes, Amand, 2012).

13. Ministerio de la Guerra (1903-1910), Resumen de la estadística sanitaria del Ejército español, Años 1903, 1904, 1905, 1906, 1907, 1908, 1909, Madrid, Imprenta del Patronato de Huérfanos de Administración Militar; (1912), Resumen de la estadística sanitaria del Ejército español, Año 1910, Madrid, Imprenta del Cuerpo de Intendencia Militar; (19131916): Resumen de la estadística sanitaria del Ejército español, Años 1911, 1912, 1914, 1915, Madrid, Imprenta del Patronato de Huérfanos de Intendencia e Intervención Militar; (1919-1920), Resumen de la estadística sanitaria del Ejército español, Años 1917, 1918. Madrid, Talleres Tipográficos "Editorial Reus" S.A.; (1921), Resumen de la estadística sanitaria del Ejército español, Año 1920, Madrid, Imprenta del Ministerio de Marina; (1929), Resumen de la estadística sanitaria del Ejército español, Año 1926, Madrid, Establecimiento Tipográfico Huelves y Compañía; (1931, 1934, 1935), Resumen de la estadística sanitaria del Ejército español, Años 1929, 1931, 1932, 1933; Madrid, Sucesores de Rivadeneyra (S.A.)-Artes Gráficas; (1932), Resumen de la estadística sanitaria del Ejército español, Año 1930, Madrid, Triunfo. Dirección General del Instituto Geográfico y Estadístico, (1915-1920), Estado de reclutamiento y reemplazo del Ejército, Años 1912-1914, 1915-1917, 1918-1920, Madrid.

14. Instituto Nacional de Estadística, Anuario Estadístico de España, Años 1888, 1915, 1916, 1917, 1918, 1919, 1920, 19211922, 1922-1923, 1923-1924, 1924-1925, 1925-1926, 1927, 1928, 1929, 1930, 1931, 1932-1933, 1934, 1936, Madrid.

15. Anónimo, (1887, 1888, 1890), “Cuadros estadísticos referentes a la asistencia prestada en los hospitales y enfermerías militares durante el año 1886", Revista de Sanidad Militar, I (12), pp. 197-198; "Cuadros estadísticos referentes a la asistencia prestada en los hospitales y enfermerías militares durante el año 1887", II (36), pp. 372-373; "Cuadros estadísticos referentes a la asistencia prestada en los hospitales y enfermerías militares durante el año 1888", IV (78), pp. 290-291. Anónimo, (1894), "Resumen de la Estadística Sanitaria del Ejército español correspondiente al año 1892", Revista de Sanidad Militar, VIII (158), pp. 25-31; (159), pp. 42-45; (160), pp. 60-64; (161), pp. 77-79, (163), pp. 108-112, (164), pp. 125-127. Larra, Ángel de (1910), "La estadística sanitaria del Ejército español en 1907", Revista de sanidad militar y la medicina militar española, IV, (10), pp. 292-297. Martínez Pacheco, Modesto (1891), "Resumen de la Estadística Sanitaria del Ejército español. Año de 1887", Revista de Sanidad Militar, V (87), pp.48-52; (88), pp. 64-68; (89), pp. 74-84. (1891), “Resumen de la Estadística Sanitaria del Ejército español. Año de 1888", Revista de Sanidad Militar, V, (90), pp. 92-97; (91), pp. 109-116; (92), pp. 126-131; (93), pp. 140-142. Potous, J. (1911), "La estadística sanitaria del Ejército español (año 1909)", Revista de Sanidad 
Militar, I, (21), pp. 669-672; (1912), "La estadística sanitaria del Ejército español (año 1910)”, II, (28), pp. 733-736; (1913): "La estadística sanitaria del Ejército español (año 1911)", III, (24), pp. 778-781; (1914): “La estadística sanitaria del Ejército español (año 1912)”, IV, (22), pp. 685-687; (1915), “La estadística sanitaria del Ejército español (año 1914)”, V, (24), pp. 753-756; (1918), "La estadística sanitaria del Ejército español (año 1916)", VIII, (10), pp. 288-291; (1919), "La estadística sanitaria del Ejército español (año 1917)", IX, (12), pp. 357359; (1920), "La estadística sanitaria del Ejército español (año 1918)", X, (14), pp. 426-428; (1921), "La estadística sanitaria del Ejército español (año 1919)”, XI, (14), pp. 418-420; (1922), "La estadística sanitaria del Ejército español (año 1920)", XII, (8) pp. 219-221; (1923), "La estadística sanitaria del Ejército español (año 1921)", XIII, (10), pp. 276-278; (1928), "La estadística sanitaria del Ejército español (año 1925)”, XVIII, (9), pp. 258-260; (1932), "La estadística sanitaria del Ejército español (año 1929)", XXII, (4), pp. 99-101. Sánchez Fernández, L. (1906), "Resumen de la estadística sanitaria del Ejército español. Año 1904", Revista de Sanidad Militar, XX, (457), pp.

\section{BIBLIOGRAFÍA}

Bernabeu Mestre, J.; Ramiro Fariñas, D.; Sanz Gimeno, A. y Robles González, E. (2003), "El análisis histórico de la mortalidad por causas. Problemas y soluciones", Revista de Demografía Histórica, XXI (I), pp. 167-193.

Blanes, Amand (2007), La mortalidad en la España del siglo XX. Análisis demográfico y territorial. Bellaterra, Universidad Autónoma de Barcelona (Tesis doctoral inédita).

Cámara-Hueso, A.D. (2006), “Fuentes documentales antropométricas en España: problemas metodológicos para los siglos XVIII y XIX", Historia Agraria, 38, pp. 115-128.

Deleito, F.G. (1902), "Algunas causas poco citadas de la mortalidad en nuestro ejército", Revista de Sanidad Militar, año XVI, no 354, pp. 109-114.

Floud, R.; Fogel, R.W.; Harris, B. Y Hong, S.C. (2011), The Changing Body. Health, Nutrition and Human Development in the Western World since 1700. NBER series, Cambridge University Press.

Gómez- Mendoza A. y Pérez-Moreda, V. (1985), "Estatura y nivel de vida en la España del primer tercio del siglo XX", Moneda y Crédito, 174, pp. 29-64.

Gómez- Mendoza A. y Pérez-Moreda, V. (1995), "Heights and Welfare in Spain, 1900-1930". En Komlos, J. (ed.), The Biological Standard of Living on Three Continents. Further Explorations in Anthropometric History, Boulder, Westview Press, pp. 81-91.

Harris, B. (1999), "Morbidity and Mortality during the Health Transition: A comment on James C, Riley, "Why Sickness and Death Rates do not Move Parallel to One Another over Time", Social History of Medicine, 1, pp. 125-131.
312-319. Torrecilla, Carlos de $(1892,1893)$ : "Resumen de la Estadística Sanitaria del Ejército español. Año de 1889", Revista de Sanidad Militar, VI, (111), pp. 33-37; (112), pp. 59-62; (113), pp. 75-78; (114), pp. 86-90; (115), pp. 106-111; (118), pp. 156-160. “Resumen de la Estadística Sanitaria del Ejército español correspondiente al año 1890", VI, (119), pp. 173-174; (120), pp. 189-192; (121), pp. 201-207; (122), pp. 221-223; (123), pp. 235-239; (125), pp. 268-272. “Resumen de la Estadística Sanitaria del Ejército español correspondiente al año 1891", VII, (133), pp. 10-15; (134), pp. 27-31; (135), pp. 44-47; (136), pp. 60-62; (137), pp. 80-84.

16. Ya en la década de 1880 se establecía una relación directa entre la incidencia de la tuberculosis y las condiciones higiénicas de los acuartelamientos, entre los que predominaban "...viejos edificios edificados con fines diametralmente opuestos al que ahora tienen, no reúnen ninguna condición higiénica..." Planter (1892: 309). Hubo que esperar a entrado el siglo XX para que las condiciones de salubridad en los cuarteles mejorases de manera significativa, Potous (1914: 685).

Johansson, S. R. (1991), "The health transition: the cultural inflation of morbidity during the decline of mortality", Health Transition Review, 1 (1), pp. 39-65.

Martínez, Felipe (1896), Memoria-resumen de la estadística sanitaria del Ejército español. Año 1894. Madrid, Imprenta del Cuerpo Administrativo del Ejército.

Martínez-Carrión, José Miguel (2014), “El bienestar de los españoles. Una historia antropométrica en perspectiva comparada", Sociologías y Economía, Universidad de Murcia. Murcia, Editum, pp. 351-368.

Martínez-Carrión, José Miguel (2012), "La talla de los europeos, 1700-2000. Ciclos, crecimiento y desigualdad", Investigaciones de Historia Económica-Economic History Research, 8 (3), pp. 176-187.

Martínez Carrión, J.M. y Pérez Castejón J.J. (2002), “Creciendo con desigualdad. Niveles de vida biológicos en la España rural mediterránea desde 1840". En: Martínez Carrión, J.M., (ed.) El nivel de vida en la España rural, siglos XVIII-XX. San Vicente, Universidad de Alicante, pp. 405-460.

Martínez Carrión, J.M. y Puche Gil, J. (2010), "La estatura de los españoles al final de la adolescencia. Una historia antropométrica comparada". En: Chastagnaret, G; Daumas, J.C.; Escudero, A. y Raveux O. (eds.), Los niveles de vida en España y Francia (siglos XVIII_XX). Publicaciones de Universidades de Alicante y de Provence, pp. 147-188.

Martínez Pacheco, Modesto (1890), Memoria-Resumen de la Estadística Sanitaria del Ejército Español correspondiente al año de 1886. Madrid, Imprenta de Jaramillo y Ca.

Murray, Christopher J.L. y Chen, Lincoln C. (1992), "Understanding Morbidity Change", Population and Development Review, 18 (3), pp. 481-503. 
Nicolau, R. (2005), “Población, salud y actividad”. En: Carreras, A. y Tafunell X. (coords.), Estadísticas históricas de España, vol I. Bilbao, Fundación BBVA, pp. 77-154.

Planter, A. (1892), "La tuberculosis pulmonar en el Ejército", Revista de Sanidad Militar, VI (128), pp. 307-311.

Pérez Moreda, V.; Reher, D. y Sanz Gimeno, A. (2015), La conquista de la salud. Mortalidad y modernización en la España contemporánea. Madrid, Marcial Pons.

Quiroga Valle, M. G. (2001), “Estatura, diferencias regionales y sociales y niveles de vida en España (1893-1954)", Revista de Historia Económica, XIX, no extraordinario, pp. 175-200.

Quiroga Valle, M. G. (2002), Medidas antropométricas y condiciones de vida en la España del siglo XX, Tesis Doctoral. Universidad de Alcalá de Henares.

Riley, J.C. (1990), "The risk of being sick: Morbidity trends in Four Countries", Population and Development Review, 6 (3), pp. 403-432.
Riley, J.C. y Alter, George (1989), "The epidemiologic transition and morbidity", Annales de Démographie Historique, Société de Démographie Historique-E.H.S.S., París, pp. 199-213.

Rodríguez-Ocaña, E. (1992), “La estadística en la administración sanitaria española del siglo veinte". En: Las estadísticas demográfico-sanitarias. Instituto de Salud Carlos III, Madrid, pp.47-77.

Sánchez Fernández, L. (1907), "El estado sanitario de los ejércitos: la mortalidad excesiva" Revista de Sanidad Militar y la Medicina Militar Española, 1 (2), pp. 42-50.

Spijker, Jeroen; Cámara, Antonio D. y Blanes, Amand (2012), "The health transition and biological living standards: Adult height and mortality in 20th-century Spain", Economics and Human Biology, 10, pp. 276-288.

Spijker, Jeroen; Pérez, Julio y Cámara, Antonio D. (2008), "Cambios generacionales de la estatura en la España del siglo XX a partir de la Encuesta Nacional de Salud", Estadística Española, 50 (169), pp. 571-604. 